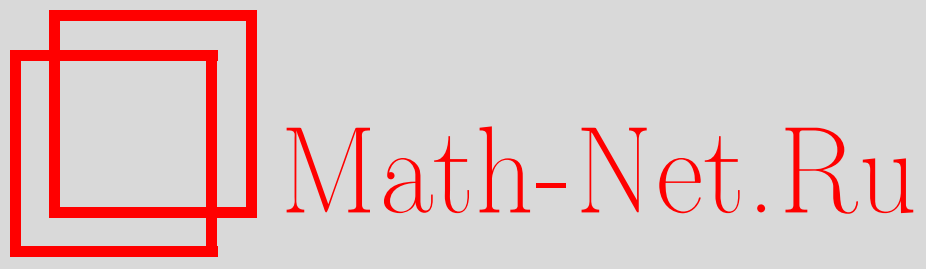

С. В. Шадрин, Некоторые соотношения на двойные числа Гурвица, Функи. анализ и его прил., 2005, том 39, выпуск 2, 91-94

DOI: https://doi.org/10.4213/faa47

Использование Общероссийского математического портала MathNet.Ru подразумевает, что вы прочитали и согласны с пользовательским соглашением

http: //www . mathnet.ru/rus/agreement

Параметры загрузки:

IP: 18.234 .156 .22

26 апреля 2023 г., 11:07:08

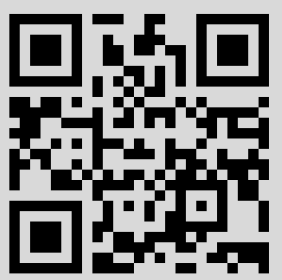


В заключение отметим, что постоянная $\gamma_{\beta}$ представляет собой естественное обобщение постоянной Эйлера $\gamma=\gamma_{1}$. Это можно увидеть, явно выписывая определяющий ряд или замечая, что $\gamma_{\beta}=\sum_{k=p+1}^{\infty} \frac{(-1)^{k}}{k} \zeta(\beta k)$. Из изложенных выше результатов следует также, что

$$
\gamma_{\beta}=\lim _{z \rightarrow 0} \frac{d}{d z} \ln z \Gamma_{\beta}(z)=\lim _{z \rightarrow 0}\left(\widetilde{R}_{\beta}(-z, 0)-\frac{1}{z}\right),
$$

в согласии с классическим случаем и независимо от функционального уравнения. С другой стороны, для произвольного $\beta$ уже неверно, что постоянная $\gamma_{\beta}$ совпадает с неособой частью соответствующей дзета-функции $\zeta_{\beta}(s, 0)$ при $s=1$.

\title{
ЛИтерАТУРА
}

1. Choi J., Quine J. R. Rocky Mountain J. Math., 26, 719-729 (1996). 2. Gilkey P. B. Invariance theorems, the heat equation, and the Atiyah-Singer index theorem. Studies in Adv. Math. CRC Press, 1995. 3. Morpurgo C. Duke Math. J., 114, 477-573 (2002). 4. Mulholland H. P. Proc. Cambridge Phil. Soc., 24, 280-289 (1928). 5. Sarnak P. Comm. Math. Phys., 110, 113-120 (1987). 6. Schuster R. Z. Anal. Anwendungen, 11, 229-236 (1992). 7. Spreafico M. Rocky Mountain J. Math., 33, 1499-1512 (2003). 8. Vardi I. SIAM J. Math. Anal., 19, 493-507 (1988). 9. Voros A. Comm. Math. Phys., 110, 439-465 (1987).

ICMC, Universidade de São Paulo, CEP 13560-970 São Carols, Brazil e-mail: mauros@icmc.usp.br
Поступило в редакцию 23 апреля 2003 г.

\section{Некоторые соотношения на двойные числа Гурвица*}

\author{
(c) 2005. С. В. ШАДРин
}

1. В этой заметке мы формулируем некоторые новые соотношения на двойные числа Гурвица. Эти соотношения приходят из теории пересечений на пространстве модулей кривых и являются прямым обобщением результатов, анонсированных в [3] и доказанных в [4].

Числа Гурвица, которые мы здесь изучаем, - это основной объект исследования в работе [2]. Мы рекомендуем работу [2], в частности, как хороший обзор по двойным числам Гурвица, содержащий наиболее полный набор ссылок по данной тематике.

2. Напомним определение чисел Гурвица. Зафиксируем целые числа $g \geqslant 0$, $n \geqslant 1$ и два неупорядоченных разбиения числа $n$ на целые положительные слагаемые, $n=a_{1}+\cdots+a_{p}=b_{1}+\cdots+b_{q}$. Рассмотрим пространство $\mathbb{C P}^{1} \mathrm{c}$ отмеченными точками $0, \infty$ и еще $2 g+p+q-2$ точками $z_{1}, \ldots, z_{2 g+p+q-2}$.

Множество мероморфных функций $f: C \rightarrow \mathbb{C P}^{1}$ степени $n$, определенных на кривых $C$ рода $g$ и таких, что $(f)=-\sum_{i=1}^{p} a_{i} x_{i}+\sum_{j=1}^{q} b_{j} y_{j}\left(x_{i}, y_{j}-\right.$

*Работа частично поддержана грантами РФФИ-01-01-00660, РФФИ-02-01-22004 и НШ1972.2002.1. 
попарно различные точки кривой $C$ ) и $z_{1}, \ldots, z_{2 g}+p+q-2-$ простые критические значения функции $f$, конечно. Здесь через $(f)$, как обычно, обозначен дивизор функции $f$.

Число таких функций называется числом Гурвица и обозначается через $H_{g}\left(a_{1}, \ldots, a_{p} \mid b_{1}, \ldots, b_{q}\right)$. При этом подсчете каждая функция $f: C \rightarrow \mathbb{C P}^{1}$ учитывается с весом $1 /|\operatorname{aut}(f)|$. Например, $H_{g}(2 \mid 2)=1 / 2$ при любом $g$, поскольку существует ровно одна гиперэллиптическая кривая $E$ и функция $f$ степени 2 на ней с фиксированным набором $2 g+2$ простых точек ветвления, причем порядок группы автоморфизмов функции $f$ равен двум.

Мы будем рассматривать только числа вида $H_{g}\left(n \mid b_{1}, \ldots, b_{q}\right)$. Для удобства введем следующее обозначение:

$$
\widehat{H}_{g, n}\left(b_{1}, \ldots, b_{q}\right):=\frac{\left|\operatorname{aut}\left(b_{1}, \ldots, b_{q}\right)\right|}{n^{2 g+q-2}(2 g+q-1) !} H_{g}\left(n \mid b_{1}, \ldots, b_{q}\right) .
$$

3. Сформулируем нашу основную теорему.

ТЕОрема 1. Для любъх $g \geqslant 0, n \geqslant 1 u b_{1}, \ldots, b_{q}, b_{1}+\cdots+b_{q}=n$, справедливо следующее соотношение:

$$
\begin{aligned}
\left(\begin{array}{l}
g \\
0
\end{array}\right) & \widehat{H}_{g, n}\left(b_{1}, \ldots, b_{q}\right)-\left(\begin{array}{c}
g \\
1
\end{array}\right) \widehat{H}_{g, n+1}\left(b_{1}, \ldots, b_{q}, 1\right) \\
& +\cdots+(-1)^{g}\left(\begin{array}{l}
g \\
g
\end{array}\right) \widehat{H}_{g, n+g}(b_{1}, \ldots, b_{q}, \underbrace{1, \ldots, 1}_{g})=\frac{(-1)^{g}}{24^{g}} .
\end{aligned}
$$

Проверим эту теорему в частном случае. Пусть $g=2$. Тогда, согласно формуле из [2],

$$
\widehat{H}_{2, n}\left(b_{1}, \ldots, b_{q}\right)=\frac{1}{24^{2}}\left(\frac{1}{2} A^{2}-\frac{1}{5} B\right),
$$

где $A=-1+b_{1}^{2}+\cdots+b_{q}^{2}, B=-1+b_{1}^{4}+\cdots+b_{q}^{4}$.

Таким образом, в этом частном случае наша теорема сводится к следующей формуле:

$$
\left(\frac{1}{2} A^{2}-\frac{1}{5} B\right)-2\left(\frac{1}{2}(A+1)^{2}-\frac{1}{5}(B+1)\right)+\left(\frac{1}{2}(A+2)^{2}-\frac{1}{5}(B+2)\right)=1,
$$

что нетрудно проверить прямым вычислением.

Формулы, аналогичные соотношению (1), существуют для произвольного рода $g$, см. [2] и [5]. Однако нам не известны никакие геометрические следствия комбинаторной структуры этих формул.

4. Объясним, как получается такое соотношение на числа Гурвица.

Рассмотрим пространство $\overline{\mathscr{M}}_{g, q+1}$ модулей кривых рода $g$ с $q+1$ отмеченными точками. Пусть $L_{1}$ - линейное расслоение над $\overline{\mathscr{M}}_{g, q+1}$ со слоем $T_{x_{1}}^{*} C$ в точке $\left(C, x_{1}, \ldots, x_{q+1}\right) \in \overline{\mathscr{M}}_{g, q+1}$. Обозначим $c_{1}\left(L_{1}\right)$ через $\psi_{1}$.

Рассмотрим подмногообразие $V_{g}^{\circ}\left(b_{1}, \ldots, b_{q}\right) \subset \mathscr{M}_{g, q+1}$, состоящее из гладких кривых $\left(C, x_{1}, \ldots, x_{q+1}\right)$, таких, что $-\left(\sum_{i=1}^{q} b_{i}\right) x_{1}+b_{1} x_{2}+\cdots+b_{q} x_{q+1}-$ это дивизор мероморфной функции. Обозначим замыкание подмногообразия $V_{g}^{\circ}\left(b_{1}, \ldots, b_{q}\right)$ в $\overline{\mathscr{M}}_{g, q+1}$ через $V_{g}\left(b_{1}, \ldots, b_{q}\right)$.

Теорема 1 непосредственно вытекает из следующих двух соотношений на пересечения на пространстве модулей кривых. 
ЛЕмма 1. Для любъх положителъных $b_{1}, \ldots, b_{q}$

$$
\begin{aligned}
\frac{(-1)^{g}}{24^{g}}= & (-1)^{g} g ! \int_{\overline{\mathscr{M}}_{g, 3}} \psi_{1}^{3 g}=\left(\begin{array}{l}
g \\
0
\end{array}\right) \int_{V_{g}\left(b_{1}, \ldots, b_{q}\right)} \psi_{1}^{2 g+q-2} \\
& -\left(\begin{array}{l}
g \\
1
\end{array}\right) \int_{V_{g}\left(b_{1}, \ldots, b_{q}, 1\right)} \psi_{1}^{2 g+q-1}+\cdots+(-1)^{g}\left(\begin{array}{l}
g \\
g
\end{array}\right) \int_{V_{g}\left(b_{1}, \ldots, b_{q}, 1, \ldots, 1\right)} \psi_{1}^{3 g+q-2} .
\end{aligned}
$$

ЛЕмма 2. Для любъх положительных $b_{1}, \ldots, b_{q}$

$$
\widehat{H}_{g, n}\left(b_{1}, \ldots, b_{q}\right)=\int_{V_{g}\left(b_{1}, \ldots, b_{q}\right)} \psi_{1}^{2 g+q-2} .
$$

Лемма 1 - это небольшое обобщение аналогичного утверждения из [3, 4], а лемма 2 - это теорема, доказанная в [4].

5. Производящая функция для рассматриваемых здесь чисел Гурвица удовлетворяет дифференциальному уравнению типа «cut-and-join» (см. аналогичные уравнения для других чисел Гурвица в $[1,6])$.

Рассмотрим функцию $F\left(\theta, x_{1}, x_{2}, \ldots\right)$, определенную равенством

$$
F\left(\theta, x_{1}, x_{2}, \ldots\right)=\sum_{g \geqslant 0} \sum_{\left(b_{1}, \ldots, b_{q}\right)} H_{g}\left(n \mid b_{1}, \ldots, b_{q}\right) \frac{\theta^{(2 g+q-1)}}{(2 g+q-1) !} x_{b_{1}} \cdots x_{b_{q}} .
$$

Теорема 2. Функиия $F=F\left(\theta, x_{1}, x_{2}, \ldots\right)$ удовлетворяет следующему уравнению:

$$
\frac{\partial F}{\partial \theta}=\frac{1}{2} \sum_{i, j \geqslant 1}\left(i j x_{i+j} \frac{\partial^{2} F}{\partial x_{i} \partial x_{j}}+(i+j) x_{i} x_{j} \frac{\partial F}{\partial x_{i+j}}\right) .
$$

Вообще говоря, эта теорема более или менее очевидна просто из геометрии разветвленных накрытий и, несомненно, имеет такое же простое комбинаторное доказательство, как аналогичная теорема в [1]. Однако эта теорема также следует непосредственно из соотношений на пересечения, полученных в [4].

В самом деле, эту теорему можно переписать как соотношение на числа Гурвица в следующем виде:

$$
\begin{aligned}
H_{g}^{\prime}\left(n \mid b_{1}, \ldots, b_{q}\right)= & \frac{1}{2} \sum_{i=1}^{q} \sum_{b_{i}^{(1)}+b_{i}^{(2)}=b_{i}} b_{i}^{(1)} b_{i}^{(2)} H_{g-1}^{\prime}\left(n \mid b_{1}, \ldots, \hat{b}_{i}, \ldots, b_{q}, b_{i}^{(1)}, b_{i}^{(2)}\right) \\
& +\frac{1}{2} \sum_{i \neq j}\left(b_{i}+b_{j}\right) H_{g}^{\prime}\left(n \mid b_{1}, \ldots, \hat{b}_{i}, \ldots, \hat{b}_{j}, \ldots, b_{q}, b_{i}+b_{j}\right),
\end{aligned}
$$

где через $H_{g}^{\prime}\left(n \mid b_{1}, \ldots, b_{q}\right)$ мы обозначаем

$$
H_{g}\left(n \mid b_{1}, \ldots, b_{q}\right) \cdot\left|\operatorname{aut}\left(b_{1}, \ldots, b_{q}\right)\right|=\widehat{H}_{g, n}\left(b_{1}, \ldots, b_{q}\right) \cdot n^{2 g+q-2}(2 g+q-1) ! .
$$

Пользуясь леммой 2, мы можем переписать соотношение (2) как соотношение на интегралы $\int_{V_{g}\left(b_{1}, \ldots, b_{q}\right)} \psi_{1}^{2 g+q-2}$. То, что мы при этом получим, будет в точности частным случаем теоремы 12.2 из [4]. 


\section{ЛитерАТУРА}

1. Goulden I. P., Jackson D. M., Vainshtein A. Ann. Comb., 4, 27-46 (2000). 2. Goulden I. P., Jackson D. M., Vakil R. arXiv: math.AG/0309440. 3. Шадрин C. B. УMH, 58, вып. 1, 197-198 (2003). 4. Shadrin S. V. Internat. Math. Res. Notices, No. 38, 20512094 (2003). 5. Shapiro B., Shapiro M., Vainshtein A. Amer. Math. Soc. Transl., Ser. 2, Vol. 180 (34), Amer. Math. Soc., Providence, RI, 1997, pp. 219-227. 6. Zvonkine D. arXiv: math.AG/0304251.

Независимый московский университет

Стокгольмский университет

Поступило в редакцию

email: shadrin@mccme.ru 6 октября 2003 г. 